\title{
SOME NETWORK MODELS RELATED TO HEAT AND MASS TRANSFER DURING THERMAL CONVERSION OF BIOMASS
}

\author{
Uldis Strautins ${ }^{1}$, Laura Leja ${ }^{2}$, Maris Gunars Dzenis ${ }^{1}$ \\ ${ }^{1}$ Institute of Mathematics and Computer Science, University of Latvia, Latvia; \\ ${ }^{2}$ University of Latvia, Latvia \\ uldis.strautins@lu.lv,1113172@edu.lu.lv
}

\begin{abstract}
The paper deals with a porous network model based simulation of effects of microwave pre-treatment of biomass pellets. Different heating regimes - rapid and slow - are compared; it is shown that rapid heating regime results in pressure build-up reaching values that cause breakage of the biomass material. Slow heating regime results in much lower maximum pressure values. As a second stage, ignition of pre-treated and non pretreated granules is compared. It is demonstrated that the pre-treated granule ignites considerably faster. The simulation considers intra-particle processes. The pellet is modelled as a porous material. The transport of volatiles is calculated using a nonlinear porous media equation. Thermal decomposition of the pellet is modelled using Arrhenius kinetics for three principal components of the biomass. An exponential rule for calculating the permeability of the material as a function of conversion rate is implemented. The model has been implemented in Octave. The result is a numerically cheap model that can be implemented and used to control the biomass gasification process. The model is versatile and can be extended to incorporate other physical and chemical processes.
\end{abstract}

Keywords: biomass, thermal conversion, pore network model, variable porosity.

\section{Introduction}

The EU 2030 climate and energy framework aims to cut greenhouse gas emissions at least by $40 \%$ of the 1990 values. In order to satisfy the increasing energy demands and simultaneously reach the aims, the framework also sets a goal to increase the usage of renewable resources in the energy sector to $32 \%$ and increase energy efficiency by 32.5\% [1]. Promoting usage of biomass as a fuel for heating purposes is a step towards this program. Latvia is an example of a country, where the biomass consumption for fuel is steadily increasing, e.g., in $201961.3 \%$ of total fuel consumption for heating purposes have been covered by biomass. This has reduced the dependency on imported energy sources from $63.9 \%$ in 2005 to $44.3 \%$ in 2018 [2].

Biomass as a source of fuel has both advantages and challenges, which explains the amount of ongoing research on biomass as a renewable energy source [3]. Certain important characteristics of biomass, such as energy efficiency, can be improved by microwave pre-treatment $[4 ; 5]$. This results in both chemical alteration of the biomass, but also in change in porosity and permeability of the biomass, which in part is accounted for by pore wall breakage due to pressure build up [4; 6]. Simulations can be employed to optimize this pre-treatment process, however, due to the complications presented by the various kinds of biomass there exists no standard method for this [7].

There is a wide array of mathematical models for biomass combustion, gasification, torrefaction $[8,9]$. Since the biomass is a porous medium, pore network models offer an alternative to continuous medium approach, see [10] for historical account and [11;12] for more recent developments. Even though this class of models allows accounting for various relevant effects in a natural way, such as anisotropy, changing porosity, changing connectivity of pore networks, heterogeneity of the material etc, these are rarely applied to model heat and mass transfer during biomass thermal conversion processes. The present paper presents such a model.

\section{Materials and methods}

We construct a porous network model with variable porosity and compressible gas flow.

Thermal decomposition of biomass is a complicated multi-stage process with various possible reaction pathways. A generally accepted approach is to separate biomass in three main components hemicellulose, cellulose and lignin, of which each decomposes into final products (volatiles, such as $\mathrm{H}_{2}$, $\mathrm{CO}$, tar, char); one bulk reaction with Arrhenius kinetics is used to model the decomposition of each component:

$$
\frac{d X_{i}}{d t}=-X_{i}^{n_{i}} A_{i} e^{-\frac{E_{i}}{R T}}
$$


where $X_{i}=\frac{M_{i}(t)}{M_{i}(0)}$

$i$ - stands either for cellulose, hemicellulose or lignin;

$n_{i}, A_{i}$ and $E_{i}$ - order of reaction, pre-exponential factor and activation energy for the corresponding species;

$R$ - gas constant;

$T-$ absolute temperature.

Such equation is solved for each vertex of the network model and for each species.

The transport of volatiles inside the biomass matrix is modelled as a flow through a porous medium with porosity dependent on the local conversion ratio, which we define as

$$
X=\frac{M}{M_{0}},
$$

where $\quad M=M_{H C}+M_{C}+M_{L}$ - total mass of the three components;

$M_{0}$ - initial mass of the biomass.

The porosity is modelled as a linear function $\phi=\phi_{0} X+\phi_{c}(1-X)$, where $\phi_{0}$ is the initial porosity of the biomass and $\phi_{c}$ is the porosity of char [13]. The local permeability $k$ is assumed to be a function of the local conversion ratio. In the present paper we will assume that the permeability changes from $10^{-11} \mathrm{~m}^{2}$ for initial biomass to $10^{-14} \mathrm{~m}^{2}$ for biomass char [7]. The results published in this paper are calculated using a simple exponential law

$$
k(X)=10^{-11-3 X} .
$$

Thermal conversion of biomass results in release of volatiles. The rates of production of the volatiles are deduced from the experimental results $[14 ; 15]$. A reasonable estimate predicts that $1 \mathrm{~g}$ of biomass releases approximately 0.51 of volatiles.

Let the total molar density of the gas mixture associated to $i$-th vertex be denoted by $n_{i}$, the total volume associated to the vertex by $V_{i}$, of which $V_{i} \phi_{i}$ are accessible to the gas. Further we denote the mass flux from $i$-th vertex to $j$-th vertex by $q_{i j}$ and the gas release rate by $R_{i}$, measured in $\mathrm{mol} \cdot \mathrm{s}^{-1}$ then the mass balance equation reads:

$$
\frac{d}{d t}\left(n_{i} V_{i}\right)=\sum_{j \sim i} n_{j} q_{j i}+R_{i}
$$

From the ideal gas law, the density can be expressed through temperature and pressure, and flux can be expressed using Darcy's law, resulting in a porous medium equation for pressure:

$$
V_{i} \frac{d}{d t}\left(\frac{P_{i} \phi_{i}}{R T_{i}}\right)=\sum_{j \sim i} \frac{k_{i j} S_{i j} P_{j}}{R T_{j} \mu}\left(\frac{P_{j}-P_{i}}{l_{i j}}+\rho_{j} g \cos \alpha_{i j}\right)+R_{i},
$$

In the expression above, $k_{i j}, S_{i j}$ denote permeability and effective cross-section area of the edge $i \sim j, \mu$ is the dynamic viscosity of the gas, $\rho g \cos \alpha$ term denotes the projection of the gravity term on the $i \sim j$ edge. If the porous medium is isotropic, $S$ scales as $l^{2}$ and $V$ scales as $l^{3}$. This equation is integrated in time with respect to the pressure field by linearization and an implicit scheme.

The heat released during the exothermal reactions is assumed to be proportional to the conversion of the species:

$$
\frac{d Q}{d t}=\beta_{H C} X_{H C}^{n_{H C}} e^{-\frac{E_{H C}}{R T}}+\beta_{C} X_{C}^{n_{C}} e^{-\frac{E_{C}}{R T}}+\beta_{L} X_{L}^{n_{L}} e^{-\frac{E_{L}}{R T}},
$$

The coefficients $\beta$ incorporate local mass of the species associated with the vertex and the enthalpies of decomposition. The heat equation involves convection, conduction and heat source terms:

$$
c_{i} \frac{d T_{i}}{d t}=\sum_{j \sim i} \gamma_{h} q_{j i}^{+}\left(T_{j}-T_{i}\right)+\sum_{j \sim i} k_{h} \frac{T_{j}-T_{i}}{l_{i j}^{2}}+\frac{d Q_{i}}{d t} .
$$

Here $c_{i}$ denotes total heat capacity of the vertex, $q_{i j}^{+}$equals the mass flux through the corresponding edge, if it is positive, or zero otherwise, and $k_{h}$ is the heat conduction coefficient of the biomass matrix. This equation is integrated in time for the temperature field by implicit scheme using an upwind discretization for the convection term. 


\section{Results and discussion}

We consider a biomass briquette subjected to microwave pre-treatment, consisting of $30 \%$ hemicellulose, $30 \%$ cellulose and $40 \%$ lignin. The simulation consists of two stages: first the pellet undergoes a microwave pre-treatment of 600s in the microwave oven, heating up to a temperature of $573 \mathrm{~K}$. We assume that the microwaves heat the briquette by a pattern reported in [4]. In the second stage, the pre-treated pellet is cooled and then ignited. We also simulate the ignition of a pellet that has not been pre-treated.

The kinetic constants are taken as follows [16]: activation energies: $E_{C}=168.61 \mathrm{~kJ} \cdot \mathrm{mol}^{-1}$, $E_{H C}=126.31 \mathrm{~kJ} \cdot \mathrm{mol}^{-1}, \quad E_{L}=87.19 \mathrm{~kJ} \cdot \mathrm{mol}^{-1}$, pre-exponential factors: $A_{C}=3.5 \cdot 10^{12} \mathrm{~s}^{-1}$, $A_{H C}=9.67 \cdot 10^{9} \mathrm{~s}^{-1}, A_{L}=2.59 \cdot 10^{5} \mathrm{~s}^{-1}$ and reaction orders: $n_{c}=1.38, n_{H C}=2.30, n_{L}=1.51$.

To model microwave pre-treatment, in which the biomass is heated to about $573 \mathrm{~K}$ in a pattern consistent with [8], it is assumed that the temperature rises from $T_{0}=373 \mathrm{~K}$ to

$$
T(x, y, z)=T_{1}+\Delta T \frac{(h-x)^{2}}{h^{2}} \cdot \frac{(h-y)^{2}}{h^{2}} \cdot \frac{(h-z)^{2}}{h^{2}},
$$

with $\quad T_{1}=543 \mathrm{~K}$ and $\Delta T=30 \mathrm{~K}$.

Two scenarios are considered: in the first scenario, the heating is rapid and happens in one time step, and in the second the temperature is raised linearly during a time period of $300 \mathrm{~s}$.

A snapshot of the results of the simulation under scenario 1 is shown in Figure 1.

a)

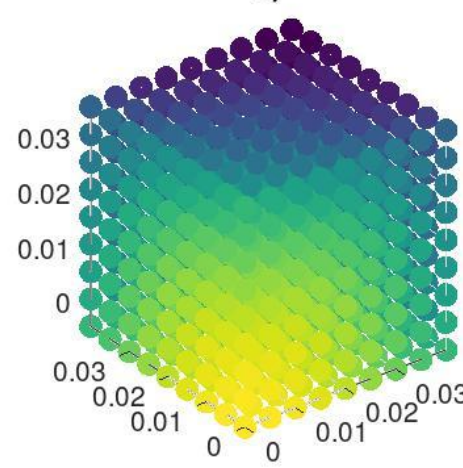

c)

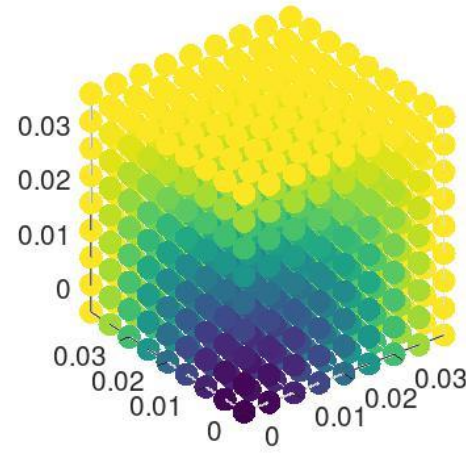

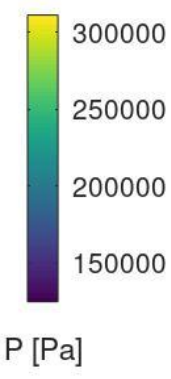

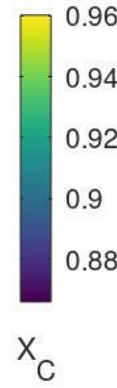

b)

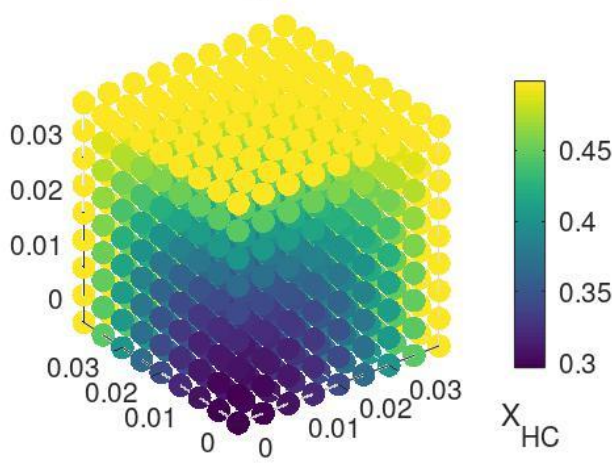

d)

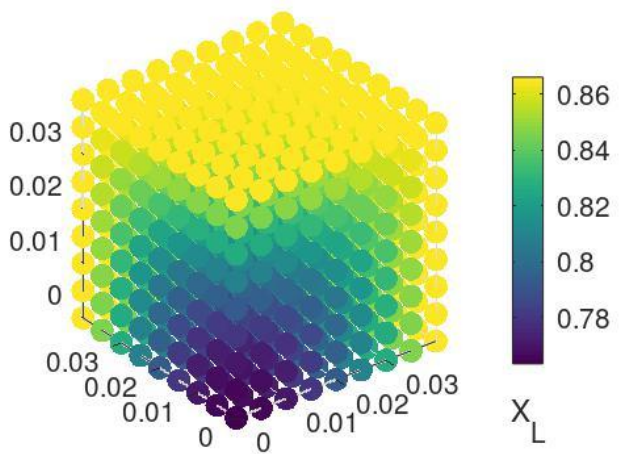

Fig. 1. Pressure at the time of reaching the highest point $(t=1.2 \mathrm{~s})(\mathrm{a})$; remaining parts of hemicellulose (b), cellulose (c) and lignin(d) at $t=200 \mathrm{~s}$; rapid heating (temperature jumps to $573 \mathrm{~K}$ )

The simulation demonstrates that the microwave pre-treatment increases the porosity and thus permeability of the biomass, mainly by decomposing part of hemicellulose (the active component with the lowest activation energy). Also, new channels between pores must be opened due to mechanical breakage caused by the pressure buildup to up to $3.27 \cdot 10^{5} \mathrm{~Pa}$ due to rapid release of volatiles and 
simultaneous increase in temperature. By the end of simulation the maximal value of pressure decays to $1.15 \cdot 10^{5} \mathrm{~Pa}$. It should be noted that similarly high increase in pressure is reported in [6].

The simulation results under scenario 2 are shown in Fig. 2. The maximum pressure reached with this scenario is $1.26 \cdot 10^{5} \mathrm{~Pa}$.

a)

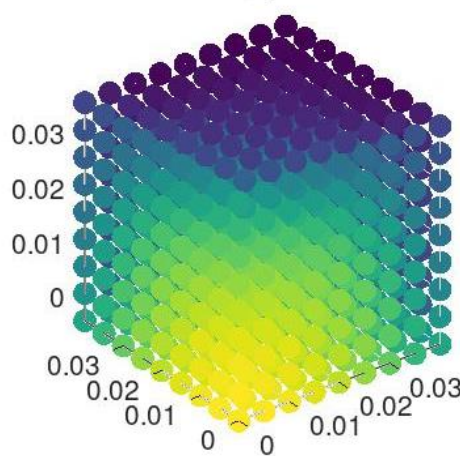

c)

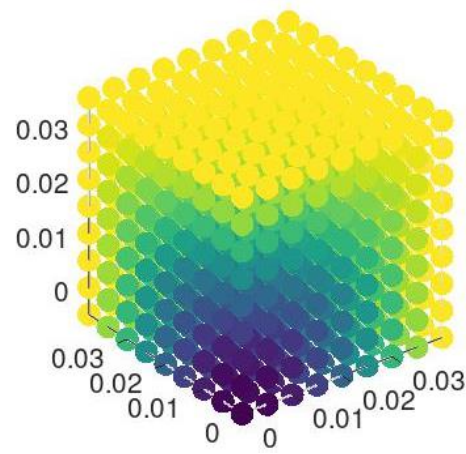

b)
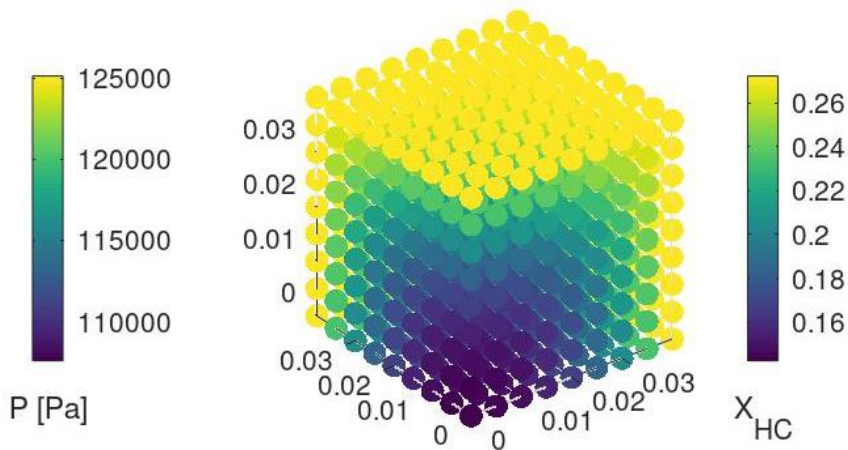

d)

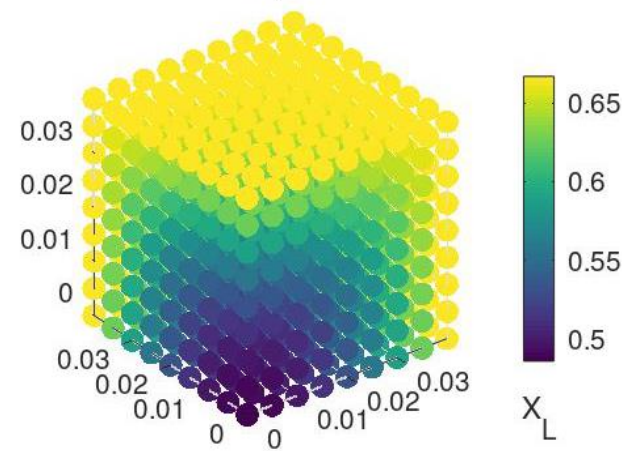

Fig. 2. Pressure (a), remaining part of hemicellulose (b), cellulose (c) and lignin(d) at $t=400 \mathrm{~s}$; slow heating (temperature rises to 573K over 300s)

We conclude that at sufficiently high heating rate there are two mechanisms that increase the permeability of the biomass sample, namely, the removal of part of the biomass matrix due to partial thermal conversion and the mechanical breakage of pore walls due to pressure buildup. At lower heating rates, only the former of these effects is at play.

Next, we investigate the ignition of an untreated pellet (Fig. 3) versus the ignition of a pre-treated pellet (Fig. 4).
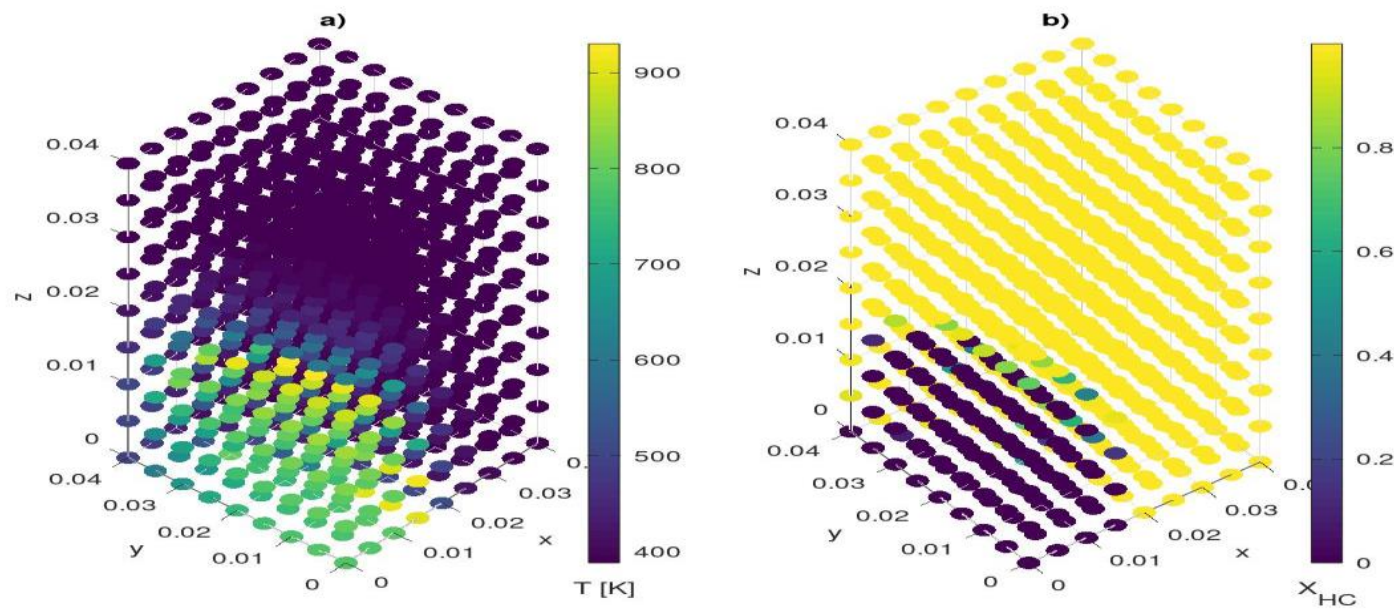

Fig. 3. Ignition of a non pre-treated pellet; temperature (a) and remaining hemicellulose (b) at time $t=300 \mathrm{~s}$ 
All other parameters are set equal in both simulations. The ignition is simulated by setting the initial temperature at the front lower corner above the temperature of ignition. A more thorough discussion of the model used is given in [18]. We see that the pre-treated pellet allows for a much stronger intraparticle convection, which results in much faster heat transfer and faster ignition. This result is in a good agreement with the experimental results reported in [5].
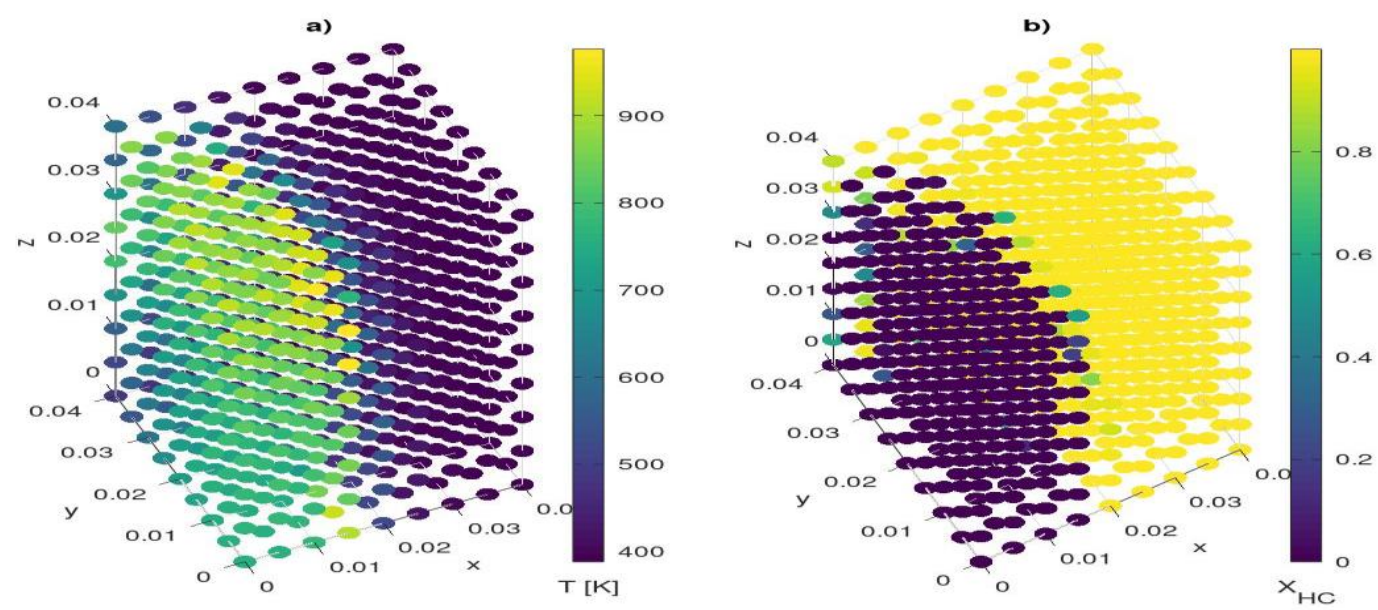

Fig. 4. Ignition of a microwave pre-treated pellet. Temperature (a) and remaining hemicellulose $(\mathrm{b})$ at $t=300 \mathrm{~s}$

\section{Conclusions}

The development and implementation of the mathematical model and the simulation results help us draw several conclusions.

1. A pore network model of intra particle heat and mass transfer during biomass thermal conversion has been developed and implemented. Numerical experiments show good qualitative agreement with the experimental results.

2. The increased efficiency of microwave pre-treated biomass granules can be explained by increased porosity and permeability of the granules. This is due to two factors: partial decomposition of the biomass (mainly hemicellulose) and pore wall breakage due to pressure buildup.

3. The maximal gas pressure inside the biomass particle depends strongly on the heating regime. Rapid heating regime leads to very high pressures that certainly lead to pore structure damage inside the particle - which happens when the pressure exceeds 2 or 3 atmospheres.

4. Increased permeability after pre-treatment results in a much faster ignition of the pellet.

\section{References}

[1] 2030 climate and energy framework. [online] [30.03.2021]. Available at: https://ec.europa.eu/clima/policies/strategies/2030_en

[2] Factsheet on wood biomass in Latvia. [online] [30.03.2021]. Available at: https://www.em.gov.lv/sites/em/files/content/fact-sheet-on-forest-biomass-in-latvia.pdf

[3] Perea-Moreno M., Sameron-Manzano E., Perea-Moreno A. Biomass as renewable energy: worldwide research trends. Sustainability vol. 11, 2019, DOI:10.3390/su11030863

[4] Kostas E.T., Beneroso D., Robinson J.P. The application of microwave heating in bioenergy: a review on the microwave pre-treatment and upgrading technologies for biomass. Renewable and Sustainable Energy Reviews, vol. 77, 2017, pp. 12-27

[5] Barmina I., Kolmickovs A., Valdmanis R., Vostrikovs S., Zake M. Thermo-chemical conversion of microwave activated biomass mixtures. IOP Conf. S. Materials Science and Engineering, vol. 355, 2018, DOI: $10.1088 / 1757-899 X / 355 / 1 / 012018$

[6] Bellais M. Modelling of the pyrolysis of large wood particles. Doctoral thesis, Stockholm, 2007. Available at: http://kth.diva-portal.org/smash/get/diva2:12258/FULLTEXT01.pdf 
[7] Fatehi H., Weng W., Li Z., Bai X, Alden A. Recent developments in numerical simulations and experimental studies of biomass thermochemical conversion. Energy Fuels, 2021, DOI: 10.1021/acs.energyfuels.0c04139

[8] Fatehi H., Bai X.S, A comprehensive mathematical model for biomass combustion. Combustion science and technology, vol. 186, 2014, pp. 574-593

[9] Popescu F., Mahu R., Ion I.V., Rusu E. A mathematical model of biomass combustion physical and chemical processes. Energies, vol. 13, 2020, DOI: 10.3390/en13236232

[10] Sahimi M., Gavalas, G.R., Tsotsis T.T. Statistical and continuum models of fluid-solid reactions in porous media. Chemical engineering science vol. 45, 1990, pp. 1443-1502.

[11] Xiong Q., Baychev, T.G., Jivkov, A.P. Review of pore network modelling of porous media: Experimental characterisations, network constructions and applications to reactive transport. Journal of Contaminant Hydrology, vol. 192, 2016, pp. 101-117

[12] Rabbani A., Babaei M., Javadpour F., A triple pore network model (T-PNM) for gas flow simulation in fractured, micro-porous and meso-porous media. Transport in Porous Media, vol. 132, 2020, pp. 707-740

[13] Di Blasi C. Modeling and simulation of combustion processes of charring and non-charring solid fuels. Prog. Energy Combust. Sci. vol. 19, 1993, pp. 71-104.

[14] Barmina I., Valdmanis R., Zake M., Ozola L., Strautins U. Development of gasification/combustion characteristics at thermo-chemical conversion of biomass mixtures. Engineering for Rural Development, 2017, pp. 54-59.

[15] Kalis H., Kolmickovs A., Marinaki M., Ozola L. Development of combustion dynamics at cocombustion of straw with wood. Engineering for Rural development, 2018, pp. 1322-1328.

[16] Dufour A., Ouartassi B., Bounaceur R, Zoulalian A. Modelling intra-particle phenomena of biomass pyrolysis. Chemical Engineering Research and Design vol. 89, 2011, pp. 2136-2146.

[17]Zhu L., Zhong Z. Effects of cellulose, hemicellulose and lignin on biomass pyrolysis kinetics. Korean Journal of Chemical Engineering, vol. 37, 2020, pp. 1660-1668.

[18] Leja L., Strautins U. A network model for thermal conversion of heterogeneous biomass granules. Engineering for Rural Development, 2021, pp. 737-743. 